\title{
Leucocyte-Rich and Platelet-Rich Fibrin(L-PRF) for the Treatment of Medication-Related Osteonecrosis of the Jaw (MRONJ) with Long Term Follow-up
}

\author{
Onur Şahin*, Toghrul Aliyev and Birkan Tatar \\ Department of Oral and Maxillofacial Surgery, Faculty of Dentistry, İzmir
}

Received: January 27, 2018; Published: February 05, 2018

*Corresponding author: Onur Sahin, Department of Oral and Maxillofacial Surgery, Faculty of Dentistry, İzmir Katip Çelebi Universty, Izmir, Turkey, Tel: 00905054410192; Email: onursahin43@hotmail.com

\begin{abstract}
Bisphosphonates (BPs), antiresorptive and antiangiogenic drugs are used to prevent metastatic bone cancers and to treat osteoporosis and Paget's disease. Besides BPs, due to the growing number of osteonecrosis cases associated with other antiresorptive and antiangiogenic therapies, American Association of Oral and Maxillofacial Surgeons (AAOMS) recommends changing the terminology of bisphosphonate related osteonecrosis of the jaws (BRONJ) and favors the term medication related osteonecrosis of the jaws (MRONJ) in 2014. The aim of this article was to report to case of a 79-year-old male with using oral ibandronate with osteonecrosis in the left retromolar area of the mandible. The therapeutic approach consisted of debridement of te osteonecrotic area and using leucocyte-rich and platelet-rich fibrin (L-PRF) for the treatment of medication-related osteonecrosis of the jaw (MRONJ).
\end{abstract}

Keywords: Antiresorptive therapy; Osteonecrosis of the jaw; Platelet-rich fibrin; Medication related osteonecrosis

Abbreviations: BPs: Bisphosphonates; AAOMS: American Association of Oral and Maxillofacial Surgeons; MRONJ: Medication Related Osteonecrosis of Jaw; BRONJ: Bisphosphonate-Related Osteonecrosis of Jaw; LLLT: Low Dose Laser Therapy; PRF: Platelet-Rich Fibrin

\section{Introduction}

Bisphosphonates (BPs) are often preferred drugs for the treatment of osteoporosis, Paget's disease and in the prevention of metastatic bone tumors. It was described in 2003 that osteonecrosis of the jaw bones is caused [1]. The increase of localized osteonecrosis cases in jaw bones related to BPs; negative effects on quality of life and increased morbidity led researchers to identify early and to investigate the effective treatment modality. It was understood that in addition to BPs, other antiresorptive and antiangiogenic drugs could cause osteonecrosis as well, so the American Association of Oral and Maxillofacial Surgeons (AAOMS) suggested to use "medication related osteonecrosis of jaw(MRONJ)" terminology instead of "bisphosphonate-related osteonecrosis of jaw (BRONJ)" [2]. In 2014, three criteria were defined by AAOMS in order to be able to perform differential diagnosis of MRONJ with many different diseases that could cause clinical osteonecrosis. These criterias are;" the presence of antiresorptive and antiangiogenic drug use in the medical history of the patient, clinical exposure of the bone site for more than 8 weeks, with no radiotherapy story or metastasis localized in the jawbone" [2].

\section{Case Report}

78-year-old male patient was referred to our clinic with complaints of halitosis, moderate and persistent pain and bone zones that were exposed in the left mandible accompanying dystrophy which arised after tooth extraction. Medical history showed that the patient used oral Ibandronate (Boniva ${ }^{\circledR}$ ) once a month due to osteoporosis for 10 years and had a tooth extraction one year before referring to our clinic. Radiologic examination revealed a poorly defined radiolucent area in the lower left wisdom tooth area and retro molar region. With the help of anamnesis, clinical and radiographic examinations, MRONJ diagnosis was made which caused by the treatment of osteoporosis with ibandronic acid. A consultation with the patient's physician was followed by a debridement of the lesion and a pathological examination after a three-month drug-free period. As a medical treatment at the end of this quarter, mouthwash $(0.12 \%$ Chlorhexidine Gluconate, Benzidamine HCL $0.15 \%$ ) with a combination of oral antibiotics Metronidazole (250 mg, 2X1) and Amoxicillin + Clavulanic acid (1000 mg, 2X1) advised for at least two weeks. 
After consultations and consents, the patient was planned to undergo intravenous sedation, inferior alveolar nerve block and local infiltration anesthesia (Ultracaine Ds $2 \mathrm{~mL}$ ) and L-PRF application after the lesion debridement. A large mucoperiosteal flap was elevated in the exposing bone tissue area. Unhealthy mucosa adjacent to exposed bone margins was removed by excisions. It was seen that the anterior left mandibular region was also affected and the site was extending posteriorly. The area of the large necrosis was excised till a firm bone surface was left and the application of L-PRF obtained from patient's blood that drawed preoperatively followed by primer wound closure. Sutures were taken on postoperative 10th day. One month after the operation, it was determined that the mucosa in the area where osteonecrosis used to present was healthy and the patient had no complaints, so followup period begun with monthly controls. Clinical and radiographic examination at the postoperative 6th month follow-up revealed healthy tissue and that the patient's symptoms had passed. After that, the patient was called once a year and no insufficient condition was seen in bone and mucosa after 4 years long follow-up.

\section{Discussion}

The treatment strategies for MRONJ, updated in 2014, have been determined by AAOMS. Conservative treatment for stage 0 and 1 , superficial debridement for stage 2, extensive debridement and resection for stage 3 were recommended [2]. Two types of treatment approaches are generally available for MRONJ treatment first of which is conservative approach, the other involves a more aggressive approach with tight closure to prevent microbial contamination after necrotic and exposed bone removal in severe cases. In the radical treatment of cases involving mandibular resection, block grants from the iliac crest accompanied with miniplate and screw systems are preferred for reconstruction of the relevant region [3]. AAOMS supports a multidisciplinary approach to patients against the risk of osteonecrosis. The benefits of early imaging and initiation of appropriate dental treatments are considerable. Not only does it reduce the incidence of MRONJ, also ensures optimal oral health for patients.

Dental imaging and appropriate dental treatment before antiresorptive and antiangiogenic treatment were observed to reduce osteonecrosis risk [4,5]. Therapeutic targets in definitive diagnosed MRONJ patients; to eliminate the pain, to control the infection in soft and hard tissues, and to minimize the necrosis of the bone that comes out. Since the publication of the 2009 guidelines, a number of studies have been published, including the results of successful treatments following operative or non-operative treatment of cases relevant with all stages of MRONJ. Although AAOMS primarily recommends conservative treatment, Ristow et al. [6] reported success rate of $20 \%$ in cases of conservative treatment, whereas it was over $85 \%$ in cases which surgical treatment decision was made. This shows that if a case with Stage 3 or cases with well-defined sequestrum is left, operative treatment should be considered as a more cautious approach when non operative treatment fails. In addition to this basic principle in the treatment protocol, ozone therapy, surgical bone grafting, stimulation of bone with growth factor, tissue engineering applications and low dose laser therapy (LLLT) are applied for supportive treatment [7-9] (Figures 1\& 2).

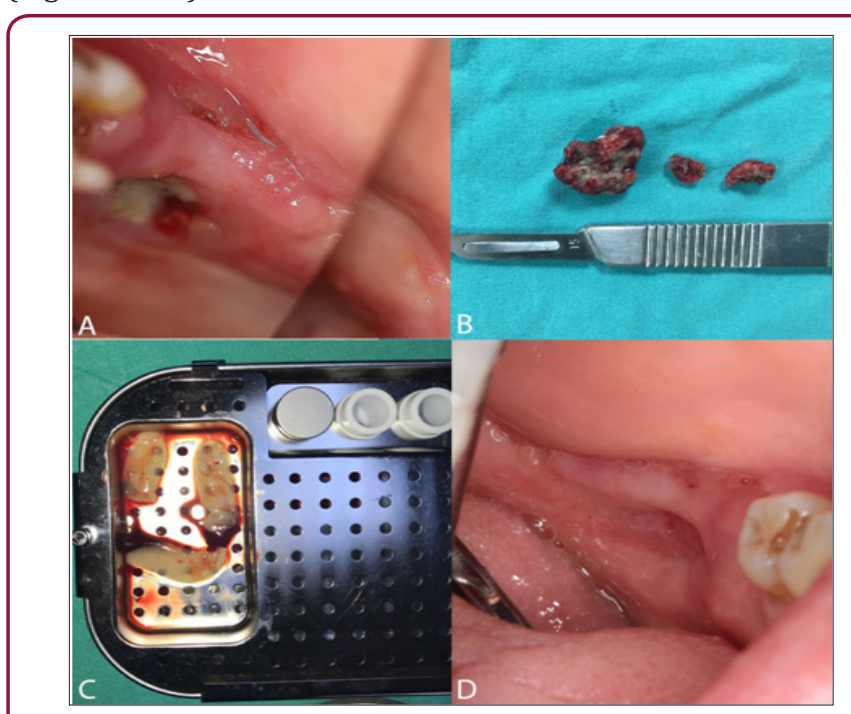

Figure 1: Intraoral photographs. A. Intraoral view showing a presence of necrotic bone exposure. B. Removal of necrotic bone debris.C. Preparation of L-PRF. D. Postop 4 years follow-up, clinical healing of the treated lesion without signs of recurrence is evident.

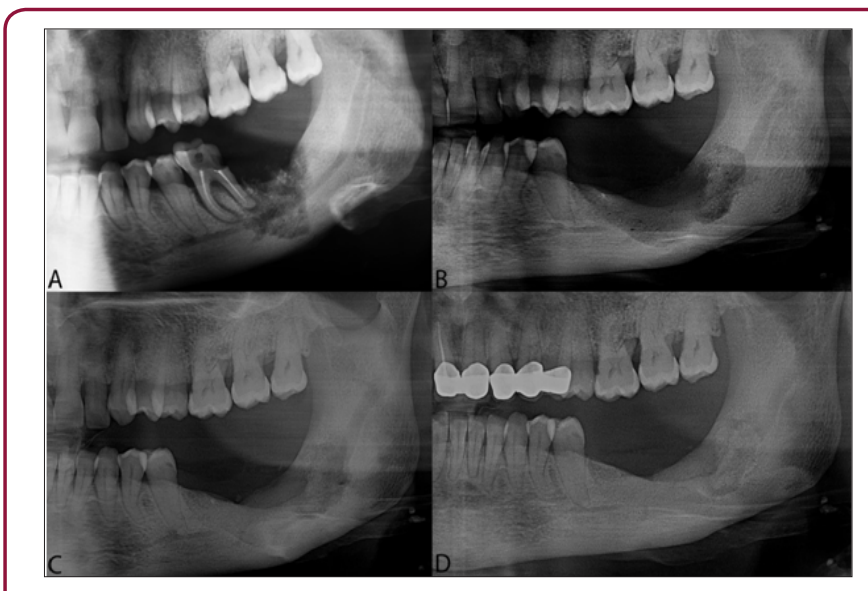

Figure 2: Panoramic radiographs A. Radiograph view demonstrating the medication related to osteonecrosis of te jaw with radiolucent area in the lower left wisdom tooth area and retro molar region B. Post-op 6 months. C. Post-op 2 years D. Post-op 4 years.

There are many studies that show success of PRF in patients with MRONJ [10]. Platelet-rich fibrin (PRF) has been shown to be beneficial in wound healing associated with bone. PRF contains angiogenic growth factors derived from alpha particles of platelets and improves bone regeneration [11]. PRF has been reported to be efficient in bone healing with angiogenic properties in patients using bisphosphonates and antiresorptive drugs [11]. We preferred to support the debrided large osteonecrosis area of Stage 2 with L-PRF by based on these approaches in the literature. No sign of pathology was seen in long-term follow-up of the patient and complete recovery was achieved. In conclusion, patients who are taking antiresorptive or antiangiogenic drugs, should be treated 
with the procedures selected by evaluating the risk factors of MRONJ, and the treatment should be performed with appropriate precautions taken. In patients with MRONJ, it is essential to have the necessary knowledge and skills in to perform the treatment carefully, and to make regular checks on the lesion that may occur again. In addition, the literature on the mechanism of action and treatment of osteonecrosis related to such drugs is still limited. Both clinical and experimental studies in which effective treatments are defined in these kinds of cases needed.

\section{References}

1. Marx RE (2003) Pamidronate (Aredia) and zoledronate (Zometa) induced avascular necrosis of the jaws: a growing epidemic. J Oral Maxillofac Surg 61(9): 1115-7.

2. Ruggiero SL, Dodson TB, Fantasia J, Goodday R, Aghaloo T, et al. (2014) American Association of Oral and Maxillofacial Surgeons on MedicationRelated Osteonecrosis of the Jaw. J Oral Maxillofac Surg 72(10): 19381956.

3. Lerman MA, Xie W, Treister NS, Richardson PG, Weller EA, et al. (2013) Conservative management of bisphosphonaterelated osteonecrosis of the jaws: staging and treatment outcomes. Oral Oncol 49(9): 977-983.

4. Yamazaki T, Yamori M, Ishizaki T, Asai K, Goto K, et al. (2012) Increased incidence of osteonecrosis of the jaw after tooth extraction in patients treated with bisphosphonates: a cohort study. Int J Oral Maxillofac Surg 41(11): 1397-1403.
5. Lazarovici TS, Yahalom R, Taicher S, Elad S, Hardan I, et al. (2009) Bisphosphonaterelated osteonecrosis of the jaws: a single-center study of 101 patients. J Oral Maxillofac Surg 67(4): 850-855.

6. Ristow O, Otto S, Troeltzsch M, Hohlweg-Majert B, Pautke C (2015) Treatment perspectives for medication-related osteonecrosis of the jaw (MRONJ). J Craniomaxillofac Surg 43(2): 290-293.

7. Ripamonti CI, Cislaghi E, Mariani, Maniezzo M (2011) Efficacy and safety of medical ozone (03) delivered in oil suspension applications for the treatment of osteonecrosis of the jaw in patients with bone metastases treated with bisphosphonates: Preliminary results of a phase I-II study. Oral Oncol 47(3): 185-190.

8. Atalay B, Yalcin S, Emes Y, Aktas I, Aybar B, et al. (2011) Bisphosphonaterelated osteonecrosis: laser-assisted surgical treatment or conventional surgery? Lasers Med Sci 26(6): 815-823.

9. Curi MM, Cossolin GS, Koga DH, Zardetto C, Christianini S, et al. (2011) Bisphosphonate-related osteonecrosis of the jaws an initial case series report of treatment combining partial bone resection and autologous platelet-rich plasma. J Oral Maxillofac Surg 69(9): 2465-2472.

10. Kim JW, Kim SJ, Kim MR (2014) Leukocyte -rich and platelet rich fibrin for the treatment of bisphosphonate-related osteonecrosis of the jaw: a prospective feasibility study. Br J Oral Maxillofac Surg 52(9): 854-859.

11. Choukroun J, Diss A, Simonpieri A, Girard MO, Schoeffler C, et al. (2006) Platelet-rich fibrin (PRF):a second-generation platelet concentrate. Part IV: clinical effects ontissue healing. Oral Surg Oral Med Oral Pathol Or al Radiol Endod 101: e56-e60.

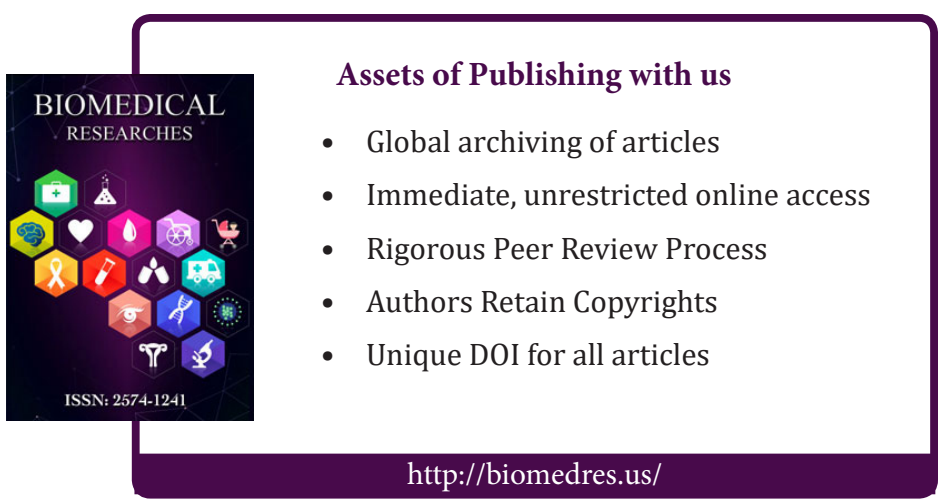

